05,06

\title{
Магнитные и магнитоэлектрические свойства ферробората $\mathrm{Tb}_{0.75} \mathrm{Ho}_{0.25} \mathrm{Fe}_{3}\left(\mathrm{BO}_{3}\right)_{4}$
}

\author{
(C) Н.В. Волков ${ }^{1,2}$, Е.В. Еремин ${ }^{1,2, \uparrow}$, В.Л. Темеров ${ }^{1}$, И.А. Гудим ${ }^{1}$ \\ ${ }^{1}$ Институт фризики им. Л.В. Киренского СО РАН, \\ Красноярск, Россия \\ ${ }^{2}$ Сибирский фредеральный университет, \\ Красноярск, Россия \\ I E-mail: eev@iph.krasn.ru
}

(Поступила в Редакцию 8 августа 2016 г.)

Групповым методом из раствора-расплава на основе тримолибдата висмута выращены монокристаллы ферробората $\mathrm{Tb}_{0.75} \mathrm{Ho}_{0.25} \mathrm{Fe}_{3}\left(\mathrm{BO}_{3}\right)_{4}$. Исследованы его магнитные и магнитоэлектрические свойства в диапазоне температур $4.2-300 \mathrm{~K}$ и в магнитных полях до 9Т. В магнитном отношении он является антиферромагнетиком с $T_{N}=38.8 \mathrm{~K}$ и анизотропией типа легкая ось. Величина магнитоэлектрической поляризации оказалась больше в 1.5-2 раза, чем сумма величин поляризации, индуцированной магнитным полем, для ферроборатов $\mathrm{TbFe}_{3}\left(\mathrm{BO}_{3}\right)_{4}$ и $\mathrm{HoFe}_{3}\left(\mathrm{BO}_{3}\right)_{4}$, взятых с соответствующими долями.

Работа выполнена при поддержке РФФИ (грант № 15-42-04186_p_сибирь_а), а также в рамках государственного задания Министерства образования и науки РФ Сибирскому федеральному университету на выполнение НИР в 2014 г. (задание № 3.2534.2014/K).

DOI: 10.21883/FTT.2017.03.44165.325

\section{1. Введение}

Тригональные редкоземельные оксибораты $\mathrm{RMe}_{3}\left(\mathrm{BO}_{3}\right)_{4}$ (где $R=\mathrm{Y}, \mathrm{La}-\mathrm{Lu} ; M=\mathrm{Fe}, \mathrm{Al}, \mathrm{Cr}, \mathrm{Ga}$, $\mathrm{Sc})$ в последние годы интенсивно исследуются многими российскими и зарубежными научными группами [1-7]. В случае боратов с двумя магнитными подсистемами (ферробораты $\left.R \mathrm{Fe}_{3}\left(\mathrm{BO}_{3}\right)_{4}\right)$ установлена их принадлежность к мультиферроикам [1,3,7]. Ферробораты имеют ромбоэдрическую структуру, описываемую пространственными группами $R 32$ или $P 3_{1} 21$. Ниже температуры Нееля $T_{N}=30-40 \mathrm{~K}$, спины ионов $\mathrm{Fe}^{3+}$ антиферромагнитно упорядочиваются, редкоземельная подсистема остается неупорядоченной вплоть до температур порядка $1 \mathrm{~K}$, однако за счет $f$-d-обмена она оказывает влияние на магнитную анизотропию ферроборатов. Ориентация магнитных моментов ионов железа относительно кристаллографических осей определяется типом редкоземельного иона: реализуется либо легкоосная антиферромагнитная структура, когда спины железа упорядочены вдоль тригональной оси $c$ $(R=\mathrm{Dy}, \mathrm{Tb}, \mathrm{Pr})[2]$, либо легкоплоскостная структура, когда спины железа упорядочены в плоскости $a b$ перпендикулярно оси $c$ кристалла $(R=\mathrm{Nd}, \mathrm{Sm}, \mathrm{Eu}$, $\mathrm{Er}, \mathrm{Y})[2]$. Кроме того, для ферроборатов $\mathrm{GdFe}_{3}\left(\mathrm{BO}_{3}\right)_{4}$ и $\mathrm{HoFe}_{3}\left(\mathrm{BO}_{3}\right)_{4}$ при изменении температуры наблюдается спин-переориентационный переход от легкоосной антиферромагнитной структуры к легкоплоскостной.

В связи с этим представляет интерес провести исследования изменения магнитной анизотропии и магнитоэлектрических свойств ферроборатов замещенных составов, в которых сочетаются два типа редкоземель- ных ионов, стабилизирующих структуры с различным типом магнитной анизотропии, что предположительно обеспечит большее разнообразие наблюдаемых эффектов и, возможно, их усиление. Например, в работе [8] показано, что максимальная величина поляризации в $\mathrm{Ho}_{1-x} \mathrm{Nd}_{x} \mathrm{Fe}_{3}\left(\mathrm{BO}_{3}\right)_{4}$ больше, чем в чистом $\mathrm{HoFe}_{3}\left(\mathrm{BO}_{3}\right)_{4}$, а в [9] экспериментальные и теоретические исследования магнитных и магнитоэлектрических свойств $\mathrm{Ho}_{1-x} \mathrm{Nd}_{x} \mathrm{Al}_{3}\left(\mathrm{BO}_{3}\right)_{4}$ позволили проанализировать возможные причины уменьшения индуцированной магнитным полем поляризации и сделать заключение о влиянии на магнитоэлектрические свойства оксиборатов со структурой хантита параметров кристаллического поля редкоземельного иона.

Настоящая работа посвящена синтезу и экспериментальному исследованию новых замещенных ферроборатов $\mathrm{Tb}_{0.75} \mathrm{Ho}_{0.25} \mathrm{Fe}_{3}\left(\mathrm{BO}_{3}\right)_{4}$, а также их сравнению с магнитными и магнитоэлектрическими свойствами $\mathrm{HoFe}_{3}\left(\mathrm{BO}_{3}\right)_{4}$ и $\mathrm{TbFe}_{3}\left(\mathrm{BO}_{3}\right)_{4}$.

\section{2. Образцы и методика измерений}

Монокристаллы $\mathrm{Tb}_{0.75} \mathrm{Ho}_{0.25} \mathrm{Fe}_{3}\left(\mathrm{BO}_{3}\right)_{4}$ выращивались из раствора-расплава на основе молибдата висмута [10] $(100-n)$ mass $\% \quad\left[\mathrm{Bi}_{2} \mathrm{Mo}_{3} \mathrm{O}_{12}+2.5 \mathrm{~B}_{2} \mathrm{O}_{33}+0.5\left[0.75 \mathrm{~Tb}_{2} \mathrm{O}_{3}\right.\right.$ $\left.+0.25 \mathrm{Ho}_{2} \mathrm{O}_{3}\right)+n$ mass $\% \quad \mathrm{~Tb}_{0.75} \mathrm{Ho}_{0.25} \mathrm{Fe}_{3}\left(\mathrm{BO}_{3}\right)_{4} . \quad$ При $n=23$ температура насыщения $T_{\text {sat }} \approx 962^{\circ} \mathrm{C}$, а еe концентрационная зависимость $d T_{\text {sat }} / d n \approx 12^{\circ} \mathrm{C} / \mathrm{mass} \%$. Кристаллы выращивались из раствора-расплава массой $120 \mathrm{~g}$ на затравках в режиме с понижением температуры $1-2^{\circ} \mathrm{C} /$ day. По завершении процесса выращивания кристаллы размером 4-6 $\mathrm{mm}$ приподнимались 
над раствором расплавом и охлаждались до комнатной температуры со скоростью не более $100^{\circ} \mathrm{C} / \mathrm{h}$.

Магнитные свойства были исследованы на вибрационном магнитометре PPMS (Quantum Design) в диапазоне температур 4.2-300 K и в магнитных полях до 9Т. Для измерения магнитоэлектрической поляризации на грани исследуемого образца, приготовленного в виде плоскопараллельной пластинки (плоскости граней перпендикулярны оси $a$ ), наносились электроды из эпоксидной смолы с проводящим наполнителем. Заряд, возникающий на образце вследствие магнитоэлектрического эффекта, измерялся электрометром Keithley 6517B. Магнитное поле прикладывалось вдоль кристаллографической оси второго порядка - оси $a-$ и вдоль оси $b$, перпендикулярной оси $a$ и оси $c$.

\section{3. Результаты и обсуждение}

3.1. С труктурный пе реход. Известно, что в ферроборатах $R \mathrm{Fe}_{3}\left(\mathrm{BO}_{3}\right)_{4}(R=\mathrm{Eu}, \mathrm{Gd}, \mathrm{Tb}, \mathrm{Dy}, \mathrm{Ho}, \mathrm{Y}, \mathrm{Er})$ co структурой хантита при понижении температуры имеет место структурный переход $R 32 \rightarrow P 3_{1} 21$, температура которого возрастает с уменьшением ионного радиуса редкоземельного иона [11]. Этот переход сопровождается аномалиями диэлектрических свойств, теплового расширения, теплоемкости, поляризации и т. п. [2]. Для ферроборатов, выращенных по технологии, используемой в данной работе, температура структурного перехода $T_{s}=92 \mathrm{~K}$ для $\mathrm{TbFe}_{3}\left(\mathrm{BO}_{3}\right)_{4}[12]$ и $T_{s}=387 \mathrm{~K}$ для $\mathrm{HoFe}_{3}\left(\mathrm{BO}_{3}\right)_{4}$ [13]. С учетом доли ионов $\mathrm{Tb}^{3+}$ и $\mathrm{Ho}^{3+} \mathrm{y}$ соединения $\mathrm{Tb}_{0.75} \mathrm{Ho}_{0.25} \mathrm{Fe}_{3}\left(\mathrm{BO}_{3}\right)_{4}$ структурный переход ожидается в районе $240 \mathrm{~K}$.

На рис. 1 представлены температурные зависимости магнитоиндуцируемой поляризации в зависимости от направления магнитного поля $\Delta P_{a a}(T)$ и $\Delta P_{a b}(T)$. Видно, что в районе $220-260 \mathrm{~K}$ на обеих зависимостях наблюдаются аномалии, которые можно связать со структурным переходом $R 32 \rightarrow P 3{ }_{1} 21$.

Совпадение расчетной температуры перехода $T_{s}$ и экспериментально определенной свидетельствует о том, что ионы $\mathrm{Tb}^{3+}$ и $\mathrm{Ho}^{3+}$ вошли в кристалл в соответствии с заданной концентрацией. Следует обратить внимание на то, что фазовый переход не является однородным и состоит из нескольких пиков. По-видимому, это вызвано неравномерностью распределения ионов $\mathrm{Tb}^{3+}$ и $\mathrm{Ho}^{3+}$ в матрице кристалла. Возможно, такая неравномерность является типичной для ферроборатов, так как, например, для $\mathrm{Nd}_{1-x} \mathrm{Dy}_{x} \mathrm{Fe}_{3}\left(\mathrm{BO}_{3}\right)_{4}$ магнитную фазовую диаграмму удалось описать только при введении двух магнитных подсистем с различающимися константами обменного взаимодействия [14].

3.2. Магнитные с в ой с ва. На рис. 2 и 3 приведены температурные и полевые зависимости намагниченности ферробората $\mathrm{Tb}_{0.75} \mathrm{Ho}_{0.25} \mathrm{Fe}_{3}\left(\mathrm{BO}_{3}\right)_{4}$, измеренные в направлении магнитного поля вдоль оси $c M_{c}(T)$, $M_{c}(H)$ и вдоль оси $a M_{a}(T), M_{a}(H)$. Из рис. 2 следует,

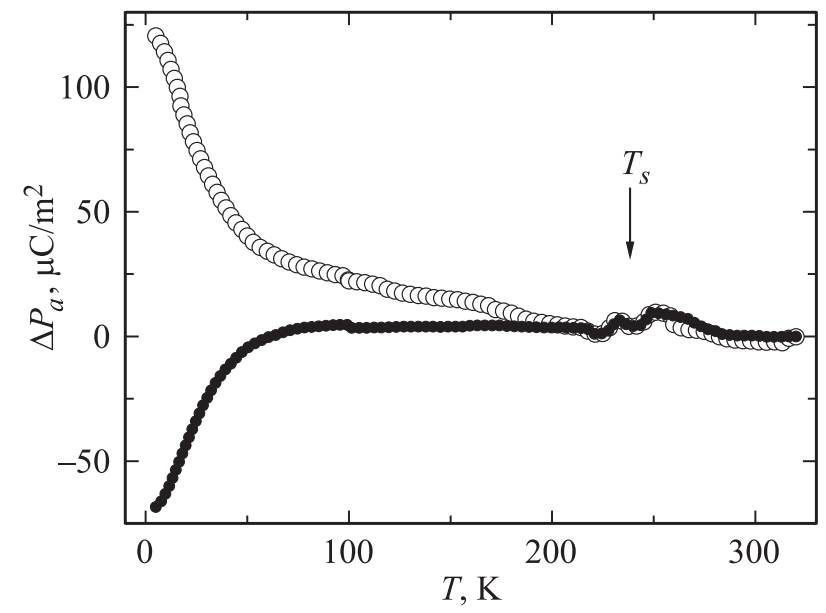

Рис. 1. Температурные зависимости продольной магнитоэлектрической поляризации $\Delta P_{a}$ вдоль оси $a$, полученные в магнитном поле 9Т в геометрии $\mathbf{B} \| \mathbf{a}$ (светлые кружки) и $\mathbf{B} \| \mathbf{b}$ (темные кружки).

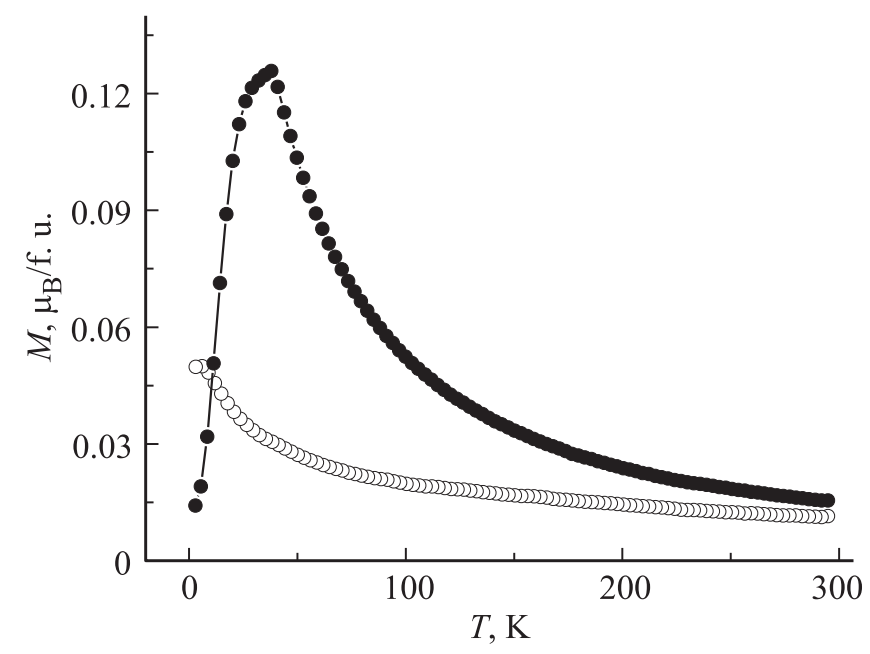

Рис. 2. Температурные зависимости намагниченности $\mathrm{Tb}_{0.75} \mathrm{Ho}_{0.25} \mathrm{Fe}_{3}\left(\mathrm{BO}_{3}\right)_{4}$, измеренные в магнитном поле $0.1 \mathrm{~T}$ в геометрии $\mathbf{B} \| \mathbf{c}$ (темные кружки) и $\mathbf{B} \perp \mathbf{c}$ (светлые кружки).

что $M_{c}(T)$ в магнитном поле $B=0.1 \mathrm{~T}$ при охлаждении от $300 \mathrm{~K}$ до $T_{N}$ возрастает по гиперболическому закону, а при $T<T_{N}\left(T_{N}=38.8 \mathrm{~K}\right)$ монотонно уменьшается и стремится к нулю при понижении температуры.

Кривая $M_{c}(T)$ по виду напоминает кривую для $\mathrm{TbFe}_{3}\left(\mathrm{BO}_{3}\right)_{4}$, т.е. вдоль направления оси $c$ магнитные свойства в основном определяются ионом Тb. Об этом свидетельствуют и полевые зависимости намагниченности $M_{c}(H)$, из которых видно, что происходит спинфлоп-переход. Такой же переход из легкоосного состояния в скошенное при направлении магнитного поля вдоль оси $c$ наблюдается и в ферроборате $\mathrm{TbFe}_{3}\left(\mathrm{BO}_{3}\right)_{4}$. Однако за счет того, что ионов $\mathrm{Tb}^{3+}$ в рассматриваемом соединении на четверть меньше, спин-флоп-переход сдвинут в область меньших полей (рис. $3, a$ ). 


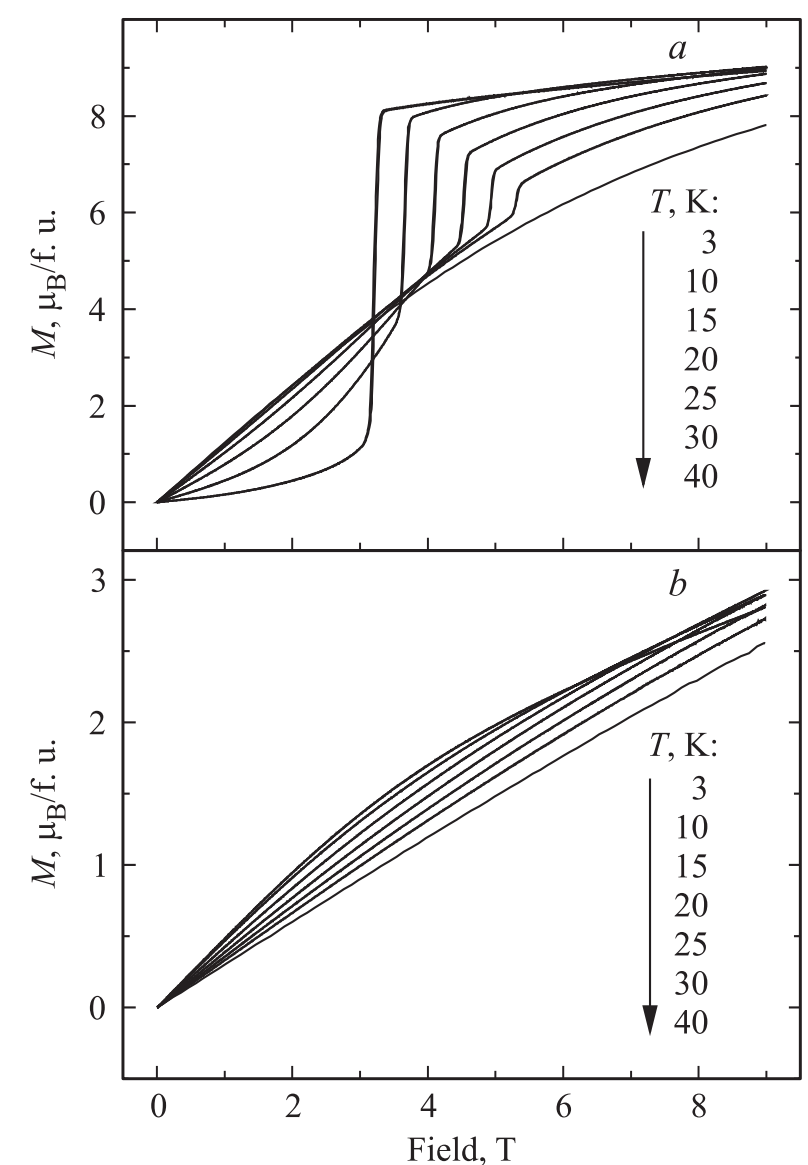

Рис. 3. Полевые зависимости намагниченности $\mathrm{Tb}_{0.75} \mathrm{Ho}_{0.25} \mathrm{Fe}_{3}\left(\mathrm{BO}_{3}\right)_{4}$, полученные при различных темпеpaтурах. $a-$ в геометрии $\mathbf{B} \| \mathbf{c}, b-$ в геометрии $\mathbf{B} \perp \mathbf{c}$.

Поскольку ион $\mathrm{Tb}^{3+}$ является сильно анизотропным (практически изинговским ионом [12]) с анизотропией типа легкая ось, влияние на магнитную анизотропию ферробората $\mathrm{Tb}_{0.75} \mathrm{Ho}_{0.25} \mathrm{Fe}_{3}\left(\mathrm{BO}_{3}\right)_{4}$ в направлении магнитного поля вдоль оси $a$ должен оказывать только ион $\mathrm{Ho}^{3+}$. Однако наблюдаемые магнитные свойства в направлении оси $a$ оказываются немного сложнее. $\mathrm{B}$ отличие от ферробората $\mathrm{HoFe}_{3}\left(\mathrm{BO}_{3}\right)_{4}$ для соединения $\mathrm{Tb}_{0.75} \mathrm{Ho}_{0.25} \mathrm{Fe}_{3}\left(\mathrm{BO}_{3}\right)_{4}$ спин-переориентационного перехода из легкоосного в легкоплоскостное состояние при повышении температуры не наблюдается, как следует из температурных и полевых зависимостей намагниченности $M_{a}(T)$ и $M_{a}(H)$ (рис. 2 и $\left.3, b\right)$.

Таким образом, можно констатировать, что в магнитном отношении ферроборат $\mathrm{Tb}_{0.75} \mathrm{Ho}_{0.25} \mathrm{Fe}_{3}\left(\mathrm{BO}_{3}\right)_{4}$ описывается в рамках модели классического двухподрешеточного антиферромагнетика с анизотропией типа легкая ось.

3.3. Магнитоэлектрические свойства.На рис. 4 представлены полевые зависимости магнитоэлектрической поляризации $\Delta P_{a a}(H)$ и $\Delta P_{a b}(H)$, полученные при различных температурах и направлениях магнит- ного поля. Видно, что величина магнитоэлектрического эффекта достигает $100 \mu \mathrm{C} / \mathrm{m}^{2}$ (при $T=5 \mathrm{~K}, B=9 \mathrm{~T}$ ), а характер кривых отличается от наблюдаемого как для $\mathrm{TbFe}_{3}\left(\mathrm{BO}_{3}\right)_{4}$, так и для $\mathrm{HoFe}_{3}\left(\mathrm{BO}_{3}\right)_{4}$. В отличие от случая $\mathrm{HoFe}_{3}\left(\mathrm{BO}_{3}\right)_{4}$ отсутствуют низкополевые особенности, связанные со спин-переориентационным переходом, индуцированным магнитным полем. Из сравнения рассматриваемого ферробората с $\mathrm{TbFe}_{3}\left(\mathrm{BO}_{3}\right)_{4}$ следует, что в нашем случае все кривые $\Delta P_{a a}(H)$ положительны, а $\Delta P_{a b}(H)$ отрицательны, тогда как в случае $\mathrm{TbFe}_{3}\left(\mathrm{BO}_{3}\right)_{4}$ это справедливо только для температур $T>50 \mathrm{~K}$. Следует обратить внимание на то, что вид полученных кривых больше всего напоминает зависимости $\Delta P_{a a}(H)$ и $\Delta P_{a b}(H)$ для парамагнитных оксиборатов со структурой хантита (см., например, [6,7]).

Что касается величины магнитоэлектрического (МЭ) эффекта, то оказывается, что простое сложение величин с соответствующими долями от ферроборатов $\mathrm{TbFe}_{3}\left(\mathrm{BO}_{3}\right)_{4}$ и $\mathrm{HoFe}_{3}\left(\mathrm{BO}_{3}\right)_{4}$ не дает совпадения с экспериментально определенными значениями.

Для направления $\mathbf{H} \| \mathbf{a}$ МЭ-эффект в $\mathrm{HoFe}_{3}\left(\mathrm{BO}_{3}\right)_{4}$ составляет примерно $\Delta P_{a a}=160 \mu \mathrm{C} / \mathrm{m}^{2} \quad(B=9 \mathrm{~T}$ и $T=5 \mathrm{~K})$ [15], а для $\mathrm{TbFe}_{3}\left(\mathrm{BO}_{3}\right)_{4} \quad \Delta P_{a a}=5 \mu \mathrm{C} / \mathrm{m}^{2}$ $(B=9 \mathrm{~T}$ и $T=5 \mathrm{~K})$ [16]. Если предположить, что

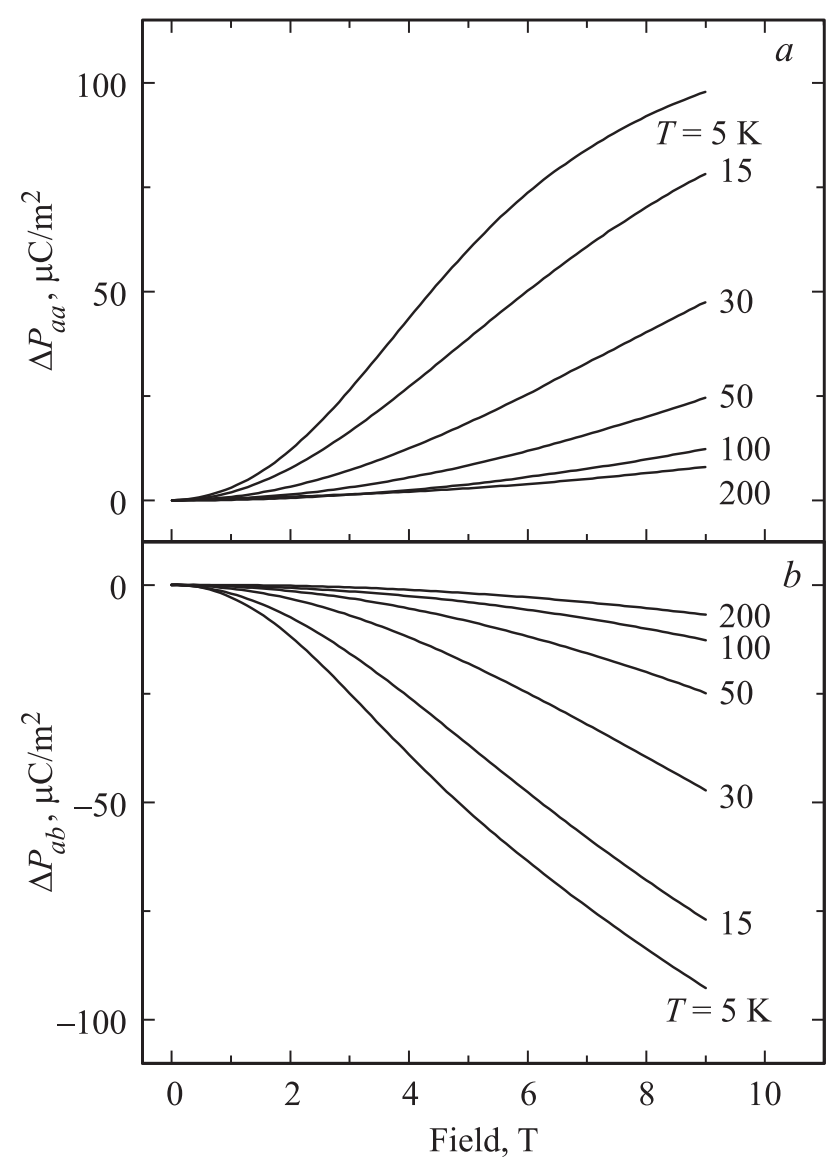

Рис. 4. Полевые зависимости продольной магнитоэлектрической поляризации $\Delta P_{a}$ при различных температурах. $a-$ в геометрии $\mathbf{B} \| \mathbf{a}, b-$ в геометрии $\mathbf{B} \| \mathbf{b}$. 
вклад в МЭ-эффект вносят ионы Но и Тb в равных долях, то для нашего соединения он должен быть равен $\Delta P_{a a}=\delta P_{a a}(\mathrm{Ho}) \cdot 0.25+\Delta P_{a a}(\mathrm{~Tb}) \cdot 0.75 \cong 45 \mu \mathrm{C} / \mathrm{m}^{2}$, что более чем в 2 раза меньше наблюдаемого в эксперименте (рис. 4, $a$ ).

Для направления $\mathbf{H} \| \mathbf{b} \quad \Delta P_{a b}\left(\mathrm{HoFe}_{3}\left(\mathrm{BO}_{3}\right)_{4}\right)=$ $=260 \mu \mathrm{C} / \mathrm{m}^{2}[15]$, a $\Delta P_{a} b\left(\mathrm{TbFe}_{3}\left(\mathrm{BO}_{3}\right)_{4}\right)=-5 \mu \mathrm{C} / \mathrm{m}^{2}[16]$. И в этом случае $\Delta P_{a b}=\Delta P_{a b}(\mathrm{Ho}) \cdot 0.25+\delta P_{a b}(\mathrm{~Tb}) \cdot 0.75$ $\cong 65 \mu \mathrm{C} / \mathrm{m}^{2}$, что меньше наблюдаемого в эксперименте примерно в 1.5 раза (рис. $4, b$ ).

Таким образом, можно констатировать, что МЭэффект в соединении $\mathrm{Tb}_{0.75} \mathrm{Ho}_{0.25} \mathrm{Fe}_{3}\left(\mathrm{BO}_{3}\right)_{4}$ оказался выше ожидаемого, что, скорее всего, связано с изменением энергетической структуры редкоземельного иона, вызванным локальными искажениями. Другими словами, параметры кристаллического поля для ионов $\mathrm{Tb}^{3+}$ и $\mathrm{Ho}^{3+}$ в соединении $\mathrm{Tb}_{0.75} \mathrm{Ho}_{0.25} \mathrm{Fe}_{3}\left(\mathrm{BO}_{3}\right)_{4}$, по-видимому, видоизменяются по сравнению с параметрами этих ионов в незамещенных ферроборатах $\mathrm{TbFe}_{3}\left(\mathrm{BO}_{3}\right)_{4}$ и $\mathrm{HoFe}_{3}\left(\mathrm{BO}_{3}\right)_{4}$. Возможно, это связано с увеличением локальных искажений в исследуемом ферроборате за счет неравномерного распределения ионов $\mathrm{Tb}^{3+}$ и $\mathrm{Ho}^{3+}$.

\section{4. Заключение}

Раствор-расплавным методом на основе тримолибдата висмута был выращен ферроборат $\mathrm{Tb}_{0.75} \mathrm{Ho}_{0.25} \mathrm{Fe}_{3}\left(\mathrm{BO}_{3}\right)_{4}$. Исследованы его магнитные и магнитоэлектрические свойства в диапазоне температур $4.2-300 \mathrm{~K}$ и в магнитных полях до $9 \mathrm{~T}$.

На температурной зависимости поляризации $\Delta P_{a}(T)$ в районе $220-260 \mathrm{~K}$ обнаружена аномалия, которую мы связываем со структурным переходом $R 32 \rightarrow P 3_{1} 21$.

В магнитном отношении $\mathrm{Tb}_{0.75} \mathrm{Ho}_{0.25} \mathrm{Fe}_{3}\left(\mathrm{BO}_{3}\right)_{4}$ является антиферромагнетиком с температурой Нееля $T_{N}=38.8 \mathrm{~K}$. Из полевых и температурных зависимостей намагниченности следует, что ферроборат хорошо описывается как классический двухподрешеточный антиферромагнетик с анизотропией типа легкая ось.

Исследование магнитоэлектрических свойств показало, что величина прямого магнитоэлектрического эффекта оказалась больше (примерно в 1.5-2 раза), чем сумма величин с соответствующими долями от $\mathrm{TbFe}_{3}\left(\mathrm{BO}_{3}\right)_{4}$ и $\mathrm{HoFe}_{3}\left(\mathrm{BO}_{3}\right)_{4}$. Скорее всего, это связано с изменением энергетической структуры редкоземельных ионов $\mathrm{Tb}^{3+}$ и $\mathrm{Ho}^{3+}$, вызванным локальными искажениями.

\section{Список литературы}

[1] А.К. Звездин, С.С. Кротов, А.М. Кадомцева, Г.П. Воробьев, А.П. Пятаков, Л.Н. Безматерных, Е.А. Попова. Письма в ЖЭТФ 81, 335 (2005).

[2] А.М. Кадомцева, Ю.Ф. Попов, Г.П. Воробьев, А.П. Пятаков, С.С. Кротов, К.И. Камилов, В.Ю. Иванов, А.А. Мухин, А.К. Звездин, А.М. Кузьменко, Л.Н. Безматерных, И.А. Гудим, В.Л. Темеров. ФНТ 36, 640 (2010).
[3] A.I. Popov, D.I. Plokhov, A.K. Zvezdin. Phys. Rev. B 87, 024413 (2013).

[4] T. Usui, Y. Tanaka, H. Nakajima, M. Taguchi, A. Chainani, M. Oura, S. Shin, N. Katayama, H. Sawa, Y. Wakabayashi, T. Kimura. Nature Mater. 13, 611 (2014).

[5] А.А. Демидов, Д.В. Волков, И.А. Гудим, Е.В. Еремин, К.Н. Болдырев. ЖЭТФ 146, 835 (2014).

[6] А.И. Бегунов, А.А. Демидов, И.А. Гудим, Е.В. Еремин. Письма в ЖЭТФ 97, 611 (2013).

[7] Н.В. Волков, И.А. Гудим, Е.В. Еремин, А.И. Бегунов, А.А. Демидов, К.Н. Болдырев. Письма в ЖЭТФ 99, 72 (2014).

[8] R.P. Chaudhury, F. Yen, B. Lorenz, Y.Y. Sun, L.N. Bezmaternykh, V.L. Temerov, C.W. Chu. Phys. Rev. B 80, 104424 (2009).

[9] Н.В. Волков, И.А. Гудим, А.А. Демидов, Е.В. Еремин. Письма в ЖЭТФ 101, 347 (2015).

[10] L.N. Bezmaternykh, V.L. Temerov, I.A. Gudim, N.A. Stolbovaya. Cryst. Rep. 50, 597 (2005).

[11] D. Fausti, A. Nugroho, P. van Loosdrecht, S.A. Klimin, M.N. Popova, L.N. Bezmaternykh. Phys. Rev. B 74, 024403 (2006).

[12] E.A. Popova, D.V. Volkov, A.N. Vasiliev, A.A. Demidov, N.P. Kolmakova, I.A. Gudim, L.N. Bezmaternykh, N. Tristan, Yu. Skourski, B. Buchner, C. Hess, R. Klinger. Phys. Rev. B 75, 224413 (2007).

[13] S.N. Sofronova, Yu.V. Gerasimova, A.N. Vtyurin, I.A. Gudim, N.P. Shestakov, A.A. Iyanenko. Vibrational Spectroscopy 72, 20 (2014).

[14] А.А. Демидов, И.А. Гудим, Е.В. Еремин. ЖЭТФ 141, 294 (2012).

[15] А.М. Кадомцева, Г.П. Воробьев, Ю.Ф. Попов, А.П. Пятаков, А.А. Мухин, В.Ю. Иванов, А.К. Звездин, И.А. Гудим, В.Л. Темеров, Л.Н. Безматерных. ЖЭТФ 141, 930 (2012).

[16] А.К. Звездин, А.М. Кадомцева, Ю.Ф. Попов, Г.П. Воробьев, А.П. Пятаков, В.Ю. Иванов, А.М. Кузьменко, А.А. Мухин, Л.Н. Безматерных, И.А. Гудим. ЖЭТФ 136, 80 (2009). 